ings of other recent conferences. The need to repeat information in this way is open to question.

In reviewing a volume of this length it is impossible to appraise separately each individual contribution, but the overall impression is that the book provides pointers to possible lines for future research rather than a body of firm facts. In an opening session the attractive concept of separate thymus and bursa dependent central immune systems is reviewed and it is concluded that the development of germinal centres is most closely related to the bursal system (or its mammalian equivalent). In this context, the centres are probably more concerned with antibody responses (mainly bursa dependent) than delayed hypersensitivity reactions (probably thymus dependent). Unequivocal evidence is presented for the localization of antigens and immunoglobulins within germinal centres, but it is not certain whether the antibody is actually produced within these sites.

Clearly, much hinges on the elucidation of the origins, functional properties and ultimate fate of lymphoid cells within the centres. It is unfortunate that in the session dealing with ultrastructural studies, some contributors attempt to draw up schemes of cellular transformations by means of the static appearances seen in tissue sections. Appropriate labelling techniques (radioisotope or chromosome marker) are always needed to determine cellular transformations with certainty. Further knowledge of the kinetics of the cells of the centres must await the successful application of these methods.

In conclusion, this book contains a valuable account of current progress in research on germinal centres. Ample evidence is provided for the importance of these centres in immune responses and in the aetiology of a number of disease processes. The centres probably result from a proliferative response to prolonged stimulation by antigen. The precise relationship of this response to antibody formation and other immunological phenomena remain problems for future study.

J. J. T. OWEN

\section{TOXICOLOGY AND TOXINOLOGY}

\section{Animal Toxins}

Edited by Findlay E. Russell. (A Collection of Papers presented at the First International Symposium on Animal Toxins, Atlantic City, N.J., USA, April 9-11, 1966.) Pp. xiii +428 . (Oxford, London and New York: Pergamon Press, Ltd., 1967.) $120 s$.

THe publication in book form of the proceedings of symposia was deplored some years ago in the pages of Nature $(193,724 ; 1962)$. This volume is described as "a collection of papers" presented at a symposium. This may mean that some contributors did not submit suitable manuscripts; it certainly means that the reader is spared the trivia and undocumented pronouncements commonly found in recorded discussions. The papers certainly have the accepted format for those appearing in the publishers' scientific journals. About 2,000 pages of their Biochemical Pharmacology costs a library $£ 35$ and a private subseriber $\mathfrak{E 5}$. The 400 pages of this bound volume cost $£ 6-$ marginal bargain for the library, but not for the individual !

There are forty-four papers dealing with the poisons produced by arthropods (eight), marine organisms (thirteen), snakes and amphibia (twenty-two), together with a final paper on the action of any of these toxins on the nerve, the muscle and the neuromuscular junction of the guineapig. The papers deal chiefly with pharmacology and toxicology (twenty), chemistry (nine), immunology (four), and biochemistry (three) of the toxins as well as the anatomy (four) of the poison glands, and zoology (three) of the poisonous animals.

It would be mere effrontery for a toxicologist to undertake a detailed critical review of this book, which embracos the subject of toxinology. In toxicology, the essential chemistry of the poison, be it synthetic or of plant origin. is usually not a problem. The fact that much less is known about the chemistry of animal poisons is not solely the result of a preference of chemists for ragwort rather than rattlesnakes as a source of their starting material but also of the innate complexity of many animal secretions. The precise modes of action of animal toxins, however, remain neither more nor less of a mystery than those of most other natural or synthetic poisons. Here toxinology and toxicology are on common ground. Meanwhile bacterial toxins will presumably remain outside the fields of both toxicology and toxinology and continue to be studied by microbiologists.

It is difficult to know what place a book such as this has in overcrowded libraries. To the general reader interested in learning something of a subject now to him or her there is too much detail in the papers. The specialist will probably have seen the detailed work reported elsewhere. As was said in 1962, there is probably a place for the recording of critical reviews and survey papers presented at symposia, and only one of this type is found in this volume.

J. M. BARNes

\section{FAILURE STORY}

Investigations into Generation $1651-1828$

By Elizabeth B. Gasking. (The History of Scientific Ideas.) Pp. 192. (London: Hutchinson and Co. (Publishers), Ltd., 1967.) 30s. net.

From the time of the Greeks until the nineteenth century the word "generation" was used for what we would now call reproduction. It included a penumbra of other subjects: genetics, embryology and the anatomy of the reproductive organs; but the central issues concerned the contribution of the sexes and the mystery of embryological development.

A number of accounts of this complex historical topic have been written, the most renowned of which is Pro. fessor Cole's Early Theories of Sexual Generation (1930). In her presentation of this subject Mrs Gasking has been concerned to avoid the pitfalls of the dramatic approachaccounts painted in the lurid colours of the clash of rival theories; and of the discovery approach - the documentation of the first discovery and first adumbration of facts and ideas which still find a place in modern biology; for she is interested in theories of generation in the seventeenth and eighteenth centuries for their own sake. She was puzzled by the naivety of supposedly eminent scientists who accepted so implausible a theory as the preformation of the germ (egg or sperm). 'This puzzlement led her to carry out the research which is described in this book.

What has her presentation to offer? First, it contains much of the work already gone over by Cole. Because Cole's book is out of print and because Gasking comes to different conclusions than he on a number of points, this part of the new book is very welcome. Her book also contains an account of the more recent work carried out by Professor Glass on Réaumur, Maupertuis and Adanson. She has also described her detailed work on Harvey and Prévost and Dumas.

Mrs Gasking holds that Harvey must not be considered as an orthodox representative of the views of the ancients on generation, but as one who broke away from their authority as a result of his observational and experimental work. Coming now to preformation, Mrs Gasking advances three reasons for the acceptance of this implausible theory. (1) That there was no plausible alternative explanation at the time. (2) That the doctrine of preformation was in harmony with the organ-ridden physiology of the day. (3) That empirical discoveries, particularly in the eight. eenth century, tended to support the theory. 